Revue des patrimoines

\title{
Patrimoine hospitalier à travers l'Europe : un dilemme entre restructuration ou désaffectation
}

\section{Pierre-Louis Laget}

\section{(2) OpenEdition}

Journals

Édition électronique

URL : http://journals.openedition.org/insitu/1903

DOI : 10.4000/insitu.1903

ISSN : 1630-7305

Éditeur

Ministère de la culture

Référence électronique

Pierre-Louis Laget, « Patrimoine hospitalier à travers l'Europe : un dilemme entre restructuration ou désaffectation », In Situ [En ligne], 3 | 2003, mis en ligne le 23 avril 2012, consulté le 19 avril 2019. URL : http://journals.openedition.org/insitu/1903 ; DOI : 10.4000/insitu.1903

Ce document a été généré automatiquement le 19 avril 2019

\section{(c) $($ i) $(9)$}

In Situ Revues des patrimoines est mis à disposition selon les termes de la licence Creative Commons Attribution - Pas d'Utilisation Commerciale - Pas de Modification 4.0 International. 


\title{
Patrimoine hospitalier à travers l'Europe : un dilemme entre restructuration ou désaffectation
}

\author{
Pierre-Louis Laget
}

1 L'Assistance publique/Hôpitaux de Paris prit l'initiative de lancer, au cours de l'année 2000 , un projet d'étude européen sur les édifices hospitaliers. Elle s'associa dans cette perspective avec l'Inventaire général (ministère de la Culture) afin d'y trouver les ressources humaines et scientifiques qui lui faisaient partiellement défaut. Plusieurs pays européens ou régions dont la Belgique (région de Bruxelles capitale), l'Espagne (Catalogne), la Grande-Bretagne, l'Italie (région d'Emilie-Romagne), les Pays-Bas, acceptèrent d'emblée de participer au projet et furent bientôt rejoints par la Finlande. Le caractère paneuropéen de cette étude permit d'obtenir une aide de la Commission européenne, avec toutefois pour contrainte d'en achever la réalisation sur une période d'une année. Vu la brièveté des délais, seules en France cinq régions furent en mesure d'y participer. Ces régions - la Bourgogne, l'Ile-de-France, le Languedoc-Roussillon, la Lorraine et le Nord-Pas-de-Calais - avaient entrepris, chacune de leur côté, une enquête thématique sur leurs hôpitaux, mais aucune n'avait été menée à terme. Une fois formulées ces restrictions portant sur le corpus étudié, tant en France qu'à l'étranger, hormis semble-t-il en Catalogne où l'inventaire tendait vers l'exhaustivité, excluaient d'emblée quelques édifices qui constituent pourtant des jalons essentiels dans l'histoire architecturale, tels « l'Ospedale maggiore » de Milan (fin du XVe siècle), l'hôpital SaintAndré de Bordeaux (1821-1829) ou encore l'hôpital mémorial franco-américain de SaintLô (1946-1956). Ce corpus offrait malgré tout un éventail de types d'édifice hospitalier extrêmement large et sa représentativité ne s'en trouvait donc guère affectée.

2 Le projet, baptisé Présent et avenir du patrimoine hospitalier européen, devait permettre, d'une part l'édition d'un guide du patrimoine hospitalier comprenant des notices descriptives d'édifices situés dans les pays ou régions concernés par l'étude et d'autre part la création d'un site Internet portant sur les divers aspects de ce patrimoine. La tenue d'un colloque à vocation internationale où seraient abordées aussi bien des 
questions sur l'histoire des hôpitaux que celles relatives à leur adaptation avec maintien de leur fonction primitive, ou au contraire à leur désaffectation et réutilisation à un usage non hospitalier. Malgré des délais bien courts, tous ces objectifs ont été atteints.

\section{Formes institutionnelles des établissements hospitaliers}

Des réunions de tous les participants, relayés par celles d'un comité scientifique restreint, furent nécessaires pour s'accorder sur une définition du patrimoine hospitalier et sur une ébauche de typologie architecturale. Deux critères qui parurent essentiels à tous les membres du comité furent retenus: l'existence, dans l'édifice considéré, d'un hébergement permanent ou à la rigueur temporaire (cas des lazarets) de caractère volontaire, et la notion de bienfaisance ou de soins visant à soulager la détresse physique, psychique, morale ou sociale des personnes hébergées. De ce fait, se trouvaient exclus d'une part toutes les institutions destinées à soulager l'une de ces détresses, mais ne comportant aucune forme d'hébergement tels qu'ateliers de charité, dispensaires, établissements de cure thermale, d'autre part celles qui hébergent éventuellement des pensionnaires, mais dont la visée est avant tout éducative telles que les écoles pour enfants pauvres dites anciennement écoles de charité, les institutions pour handicapés de naissance - instituts de jeunes aveugles, instituts de sourds et muets - , enfin celles où s'exerce certes une certaine forme de bienfaisance, mais sur une population ne présentant aucune détresse particulière telles que crèches, colonies de vacances, maisons de retraite, etc., que l'on pourrait ranger dans les édifices sociaux.

Parmi les institutions de bienfaisance qui posaient difficulté, se trouvaient certains établissements pour enfants, notamment ceux désignés à l'époque moderne sous le nom d'orphelinats. En effet, dans la forme moderne de cette institution, l'aspect éducatif prime et ces orphelinats ne se différencient guère d'autres établissements scolaires alors que, sous l'Ancien Régime, de nombreuses maisons connues sous des noms divers hospice, maison de charité, etc. - avaient été tout spécialement fondées pour recueillir les orphelins et enfants abandonnés afin d'assurer d'abord leur survie, secondairement leur éducation. Après discussion et de manière un peu arbitraire, les formes modernes d'orphelinat furent finalement exclues de la définition du patrimoine hospitalier, tandis que les formes anciennes étaient conservées. Par ailleurs, les hôpitaux généraux (et autres institutions semblables, "workhouses" anglais, "alberghi dei poveri» italiens, " casas de misericordia » espagnoles, "spinhuis " et "rasphuis» hollandais, etc.), fondés initialement avec une certaine intention répressive, mais détournés à des fins essentiellement charitables, furent, après quelques hésitations, inclus dans le champ de l'étude.

5 Ces interrogations sur ce qui relève ou non du patrimoine hospitalier résultent principalement du fait que l'on se trouve en présence d'établissements qui étaient à l'origine, sous des dénominations variables - hôpital, hospice, hôtel-Dieu, maison-Dieu, etc. - avant tout des fondations charitables, et dont les bâtiments, parfois vieux de plusieurs siècles, demeurent toujours en usage, alors que la fonction qu'ils abritent a considérablement évolué, surtout depuis le milieu du XIX siècle. En effet, l'exercice de la médecine à l'hôpital, demeuré très longtemps marginal, prit véritablement son essor à la fin du XVIII ${ }^{e}$ siècle, notamment avec la fondation, dans les grands hôpitaux, d'écoles 
cliniques (enseignement de la médecine au lit du malade). Ainsi le soin proprement médicalisé devint progressivement prééminent dans le courant du XIX ${ }^{e}$ siècle, reléguant au second plan la fonction caritative traditionnelle. La scission de caractère institutionnel introduite en France, au milieu du XIX siècle, entre d'un côté les hôpitaux (accueil des indigents malades), de l'autre les hospices (hébergement des gens - vieillards, infirmes, orphelins - incapables d'assurer leur subsistance) ne fit qu'accélérer une médicalisation qui devait déboucher, au milieu du XX $\mathrm{XX}^{\mathrm{e}}$ siècle, sur l'ouverture de l'hôpital aux malades non indigents.

6 Le divorce actuel entre institutions à vocation sociale et établissements de soins médicalisés rendait extrêmement ardue, voire impossible, toute définition de caractère général pour des termes portant sur des édifices ayant évolué de manière aussi radicale en conservant leur nom originel. En outre, lorsqu'on fut amené à changer une désignation, ce fut simplement pour tenter de masquer, par une simple substitution de terme, la réalité d'un type d'établissement discrédité : ainsi pendant la Révolution, on proscrit du langage officiel à la fois les termes hôpital et hôtel-Dieu, l'institution ellemême ayant été décriée par les philanthropes, pour leur substituer celui, plus neutre, d'hospice, avant que ce terme lui-même ne subisse de nos jours pareil ostracisme à cause des connotations péjoratives dont il s'était entre-temps chargé. On trouve le même cas de figure avec les asiles d'aliénés rebaptisés hôpitaux psychiatriques, puis centres hospitaliers spécialisés, encore une nouvelle fois débaptisés à une date toute récente.

\section{Définition d'une typologie}




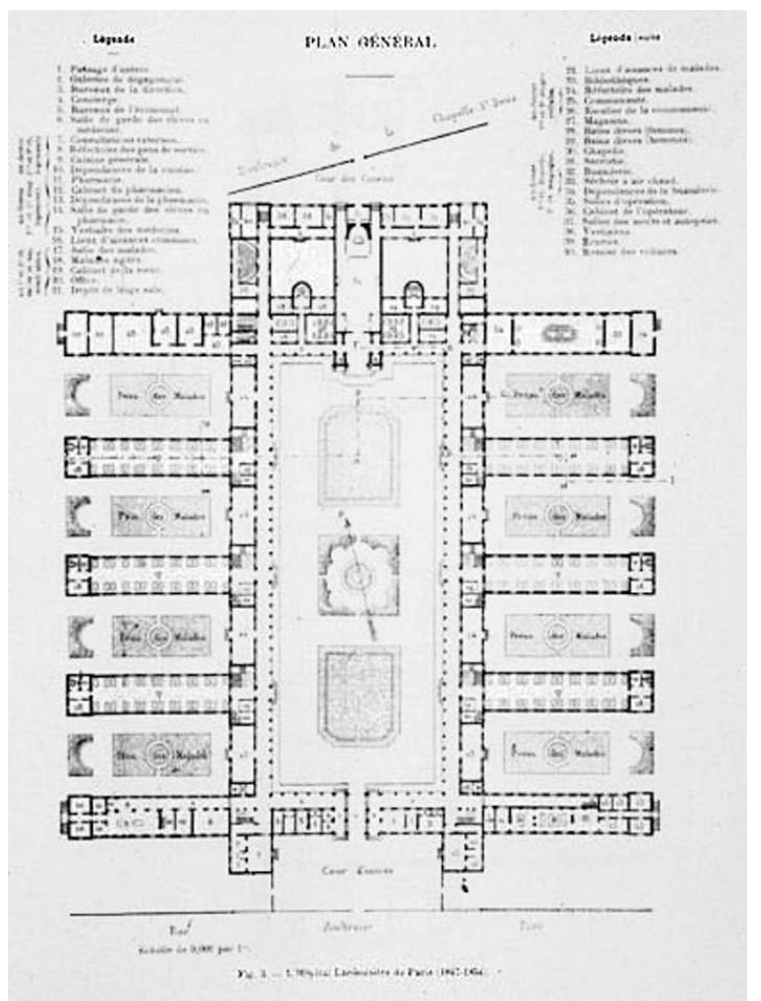

Paris, hôpital de Lariboisière. Plan de distribution générale du rez-de-chaussée. Dans : Tollet, C. LES HÔPITAUX MODERNES AU XIXE SIÈCLE. DESCRIPTION DES PRINCIPAUX HÔPITAUX FRANÇAIS ET ÉTRANgERS LES PLUS RÉCEMMENT Édifiés. Paris, 1894. Fig. $n^{\circ} 3$

Phot. Inv. P. Thibaut ( $)$ Inventaire général, ADAGP, 2003

7 Si la liste des différentes formes institutionnelles d'hôpital, avec ses équivalents dans chacune des langues des pays participants, put être dressée sans trop de difficultés, l'adoption d'une typologie fut en en revanche l'objet de discussions beaucoup plus serrées au sein du comité scientifique. Pourtant, contrairement aux institutions, variables d'un pays à l'autre, les formes architecturales avaient, selon l'avis général, évolué en parcourant exactement les mêmes étapes, et ce presque au même rythme. Les divergences de point de vue sur la définition de la typologie transcendaient d'ailleurs largement les appartenances nationales et paraissaient relever d'une différence de vision entre des historiens qui, par leurs recherches, avaient jusqu'alors plutôt abordé l'étude d'hôpitaux édifiés avant le XXe siècle, et des architectes qui étaient plutôt enclins, par leur formation, à privilégier tout particulièrement ceux du $\mathrm{XX}^{\mathrm{e}}$ siècle, $\mathrm{y}$ compris les plus récemment conçus. Cette connaissance plus fine de l'évolution architecturale du siècle en question amena les architectes à y multiplier les distinctions typologiques et à négliger, sinon à réduire, celles des époques antérieures. 
Figure 2

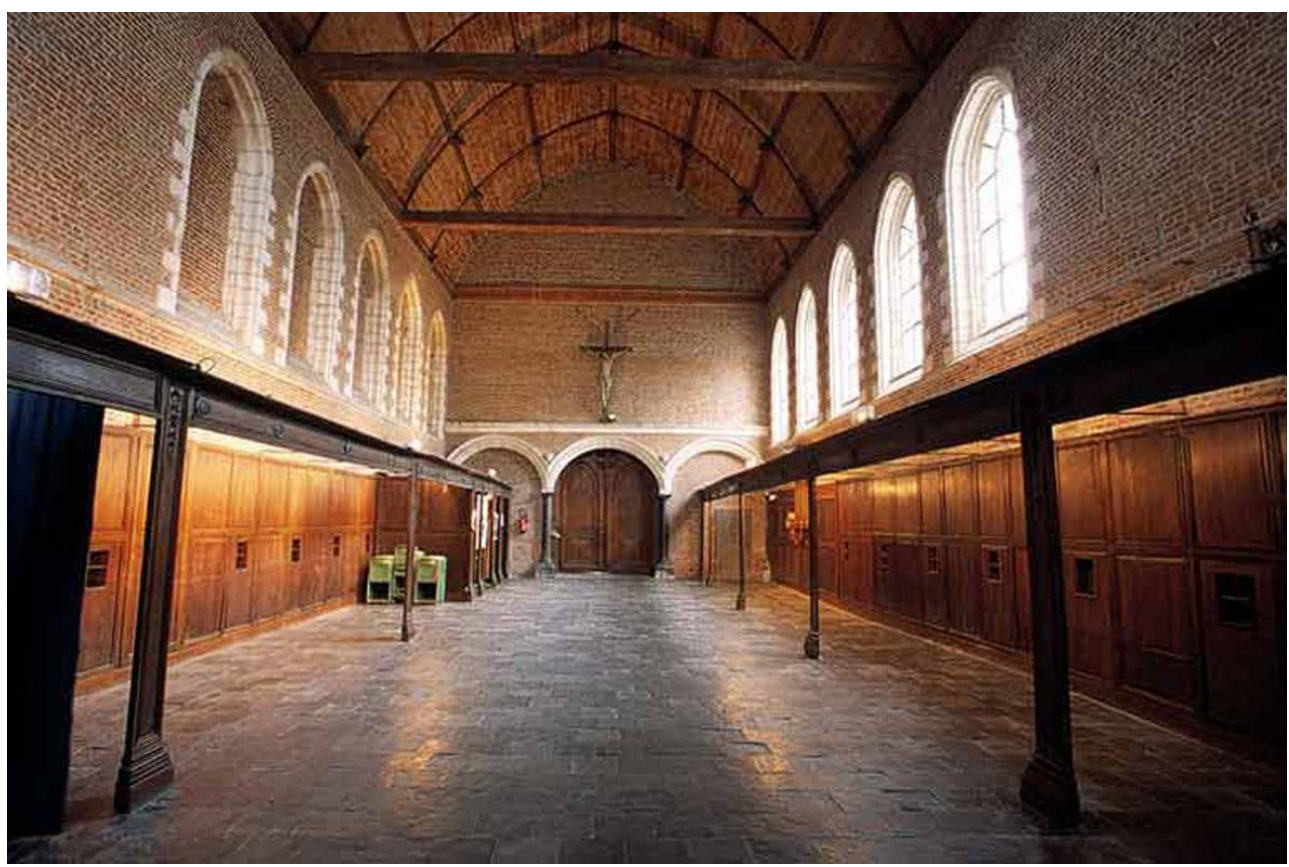

Seclin, hôpital-hospice dit Centre Marguerite de Flandre. Grande salle des malades dite salle SaintRoch, vue intérieure en regardant vers le mur de séparation entre la salle et la chapelle

Phot. Inv. P. Thibaut @ Inventaire général, ADAGP, 2001

Les désaccords entre « architectes » et « historiens ", particulièrement sur l'importance à accorder ou non à la distribution autour d'une cour ouverte ou fermée, n'empêcha pas de mettre au point une typologie qui constitue une des informations majeures dans la notice descriptive ou fiche analytique que l'on rédigerait sur chacun des édifices étudiés. Afin de rendre compte de la façon la plus précise possible, des changements de parti architectural, certains termes définissant traditionnellement des types hospitaliers furent restreint dans leur acception et la réalité qu'ils recouvraient, scindée en plusieurs entités distinctes. Ainsi le plan qualifié de pavillonnaire, se trouvait scindé, d'un commun accord, en deux entités distinctes selon que les bâtiments étaient ou non reliés par des galeries: les hôpitaux à bâtiments parallèles reliés entre eux, comme par exemple à l'hôpital Lariboisière, seraient rangés dans la catégorie des édifices de plan en peigne, et seuls les hôpitaux à bâtiments isolés seraient qualifiés de pavillonnaires. De même, le type désigné comme hôpital en hauteur, lequel supplanta l'hôpital pavillonnaire dans les années 1930, fut décliné en trois variétés successives, ce en fonction du regroupement ou non des services généraux et du plateau technique et la répartition des étages dévolus à l'hospitalisation. Ces distinctions en apparence séduisantes, portant sur une période très contemporaine de l'histoire architecturale, ne furent guère contestées, la période étant fort mal connue de la majorité des participants. 


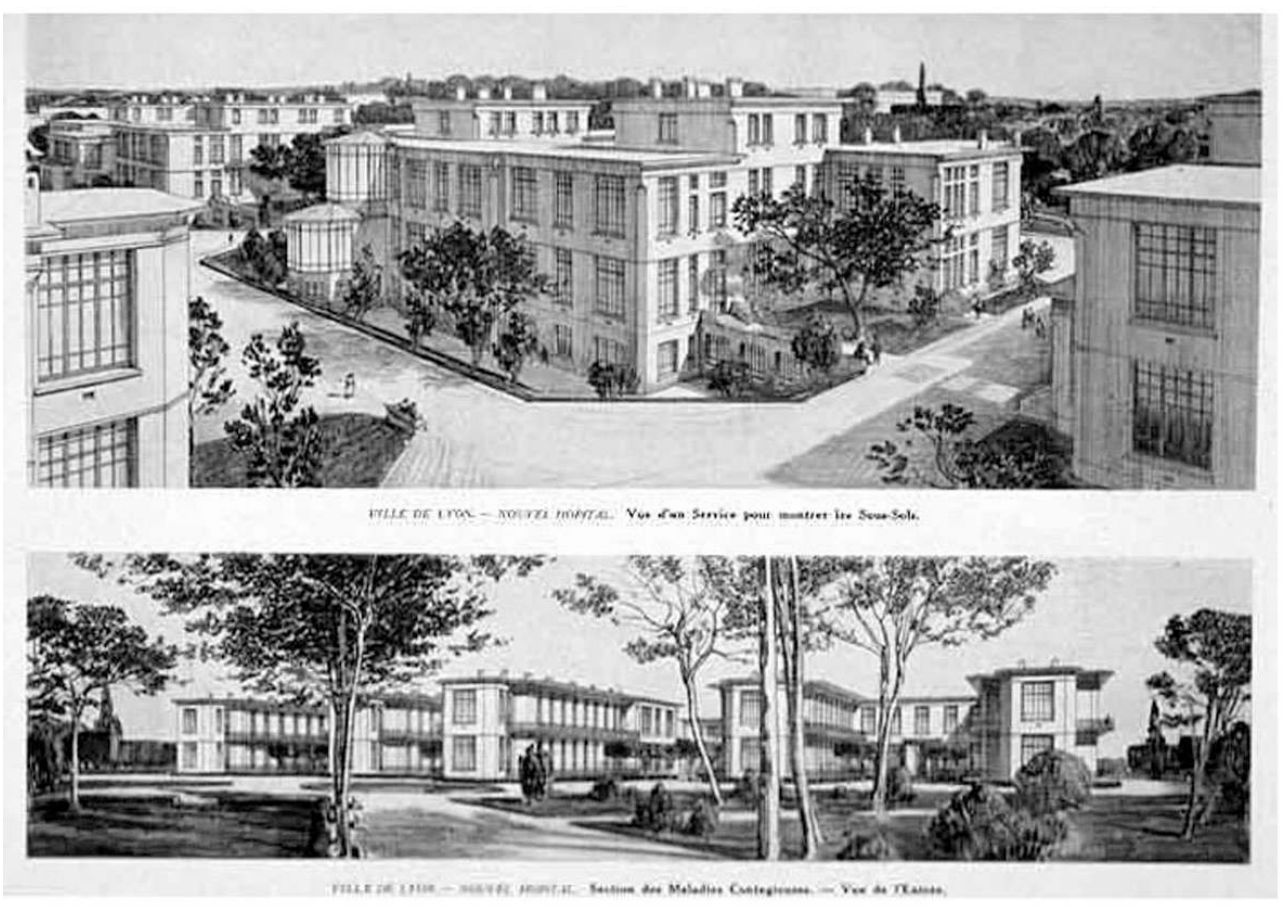

Lyon, hôpital de Grange-Blanche, baptisé depuis sous le nom d'Edouard Herriot. Vue cavalière d'un pavillon de chirurgie, destinée à montrer son étage de soubassement, et vue de l'élévation, du côté de l'entrée, du groupe de bâtiments abritant la section des maladies contagieuses ; plan gravé. Dans : Garnier, Tony. VILLE DE LYON, PROJET DE CONSTRUCtion D'UN NOUVEL HÔPITAL À GRANgE-BLANCHE. [Lyon], [1911]

Phot. Inv. P. Dapvril @ Inventaire général, ADAGP, 2002

En fin de compte, une première liste de sept types fut arrêtée. Cette liste comportait, dans l'ordre chronologique d'apparition des types : hôpital type halle, tel l'hospice de Seclin, hôpital en damier, tel l'hôpital Saint-Louis à Paris, hôpital en peigne, tel l'hôpital SaintAndré de Bordeaux, hôpital pavillonnaire, tel l'hôpital de Grange-Blanche ou EdouardHerriot à Lyon, hôpital monobloc tel le centre hospitalier de Lille, hôpital tour sur socle tel l'hôpital mémorial franco-américain de Saint-Lô, hôpital polybloc tel le nouvel hôpital Bichat à Paris. 
Figure 4

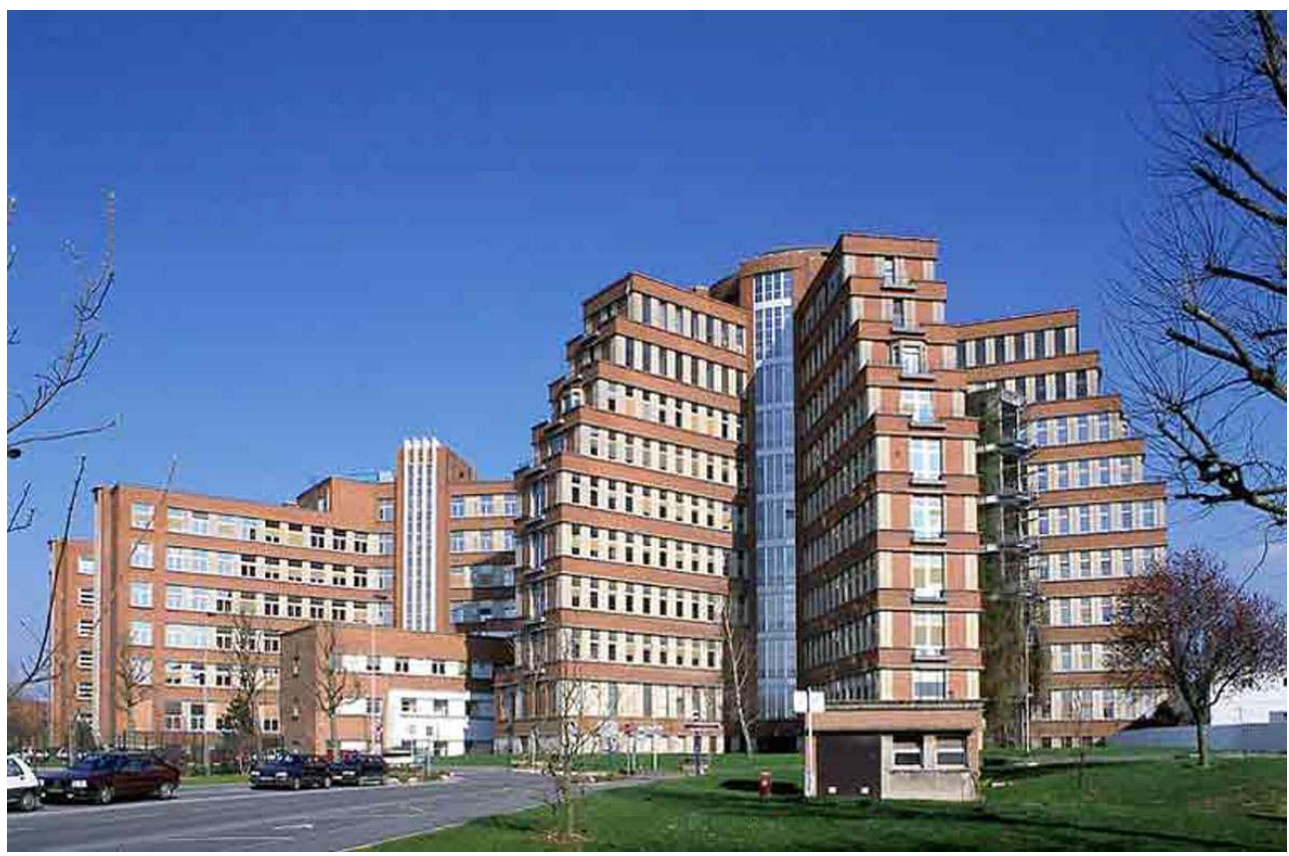

Lille, ensemble formé par l'hôpital Claude Huriez et la Faculté de médecine dit centre hospitalier régional universitaire. Vue générale prise de l'ouest montrant le bâtiment d'hospitalisation de plan en étoile sis à l'ouest et l'élévation latérale gauche de la Faculté de médecine

Phot. Inv. P.L. Laget @ Inventaire général, ADAGP, 1999

Des aberrations relatives à la désignation typologique, apparues à la lecture des premières fiches descriptives adressées au siège de l'Assistance publique à Paris (ainsi le terme hôpital en hauteur était appliqué à d'humbles édifices hospitaliers, de plan rectiligne, élevés seulement de deux ou trois étages), conduisirent finalement les « architectes » à introduire un huitième, et même un neuvième type. 


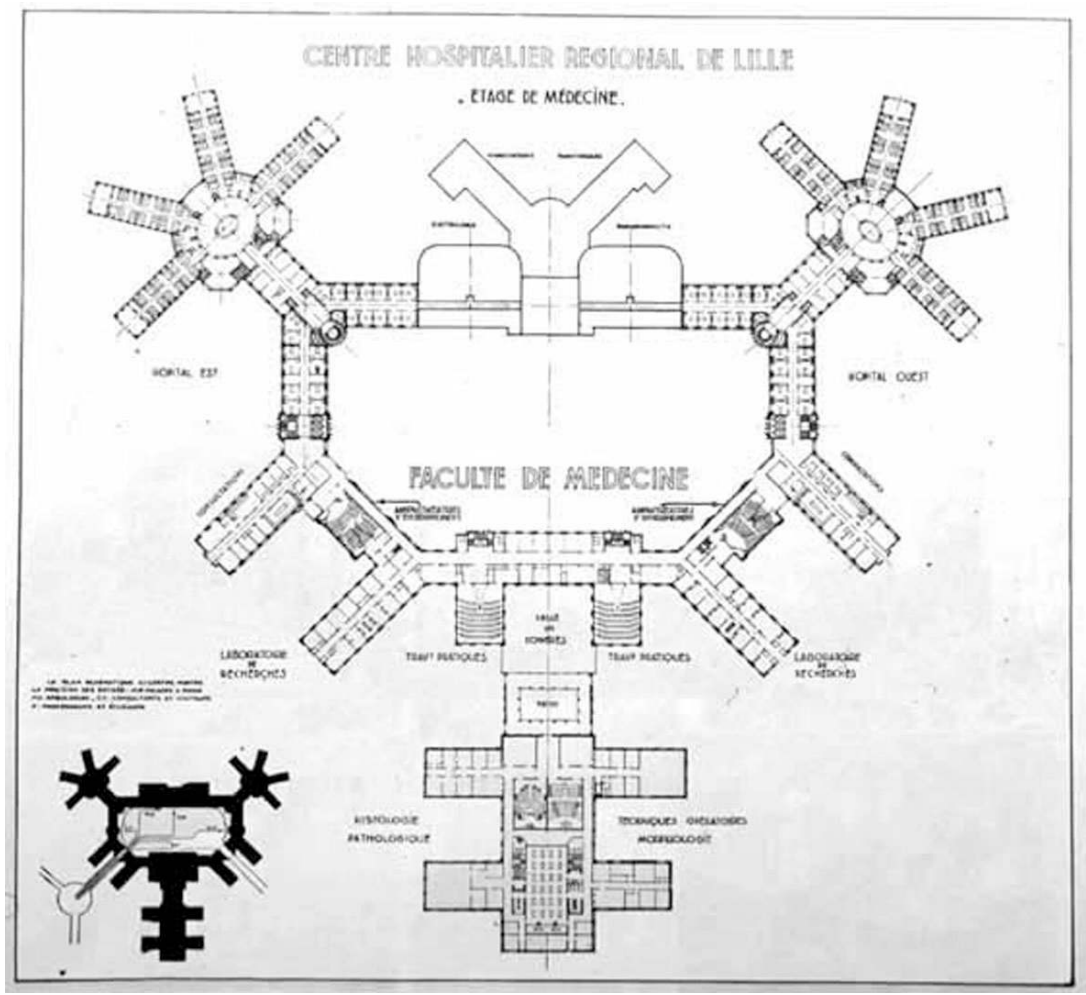

Lille, ensemble formé par l'hôpital Claude Huriez et la Faculté de médecine dit centre hospitalier régional universitaire. Plan de distribution d'ensemble avec la distribution du rez-de-chaussée de la faculté de médecine, celle du 4e étage de l'hôpital. Dans : Swynghedauw, P., Huriez, C. « L'hôpital régional de Lille - Centre hospitalier universitaire de la région du Nord ». TECHNIQUES HOSPITALIÈRES ET SOCIALES, 1959, juin. P. 20

Phot. Inv. P. Thibaut @ Inventaire général, ADAGP, 2000

11 Au seul hôpital monumental à cour fermée, comme l'hôpital Saint-Louis de Paris, serait désormais appliqué le terme d' « hôpital en damier ", tandis que l'hôpital à cour ouverte, comme l'hôtel-Dieu du Creusot, constituerait une nouvelle entité désignée sous le nom de "palais hospitalier». En outre fut introduit le terme «maison hospitalière » pour désigner de modestes établissements sans véritable caractère architectural, ayant l'aspect de banales demeures urbaines.

\section{Guide du patrimoine hospitalier}

12 A partir de l'ensemble des fiches envoyées par les différents pays, les participants au projet se concertèrent pour effectuer une première sélection à raison de 8 à 10 édifices pour chacun des sept pays, en tenant compte de l'éventail des types architecturaux, et des fonctions propres à chaque établissement. Cette sélection devait permettre de constituer un corpus d'édifices remarquables représentatif des établissements dans leur diversité, destiné à illustrer un guide du patrimoine hospitalier. A partir de cette sélection initiale, 45 édifices furent retenus pour figurer dans ce guide ${ }^{1}$. En outre, l'ensemble des fiches, au nombre de plus de 200 actuellement, allait servir à constituer un site web créé dans le cadre du projet. Ce site comporterait, outre les fiches analytiques, illustrées au moins par un plan et une ou deux vues de l'édifice, une section documentaire comprenant un 
glossaire relatif aux dénominations des divers établissements hospitaliers, une définition des types architecturaux et une bibliographie générale.

Les services des l'Assistance publique qui assurèrent la logistique de ces diverses opérations - élaboration du guide, mise en place du site, organisation du colloque réussirent à respecter l'échéancier et à faire en sorte que le colloque, qui se tint à Paris les 13 et 14 décembre 2001, fût irréprochable quant à son organisation, tout en étant d'un excellent niveau scientifique.

Quoique l'aide de la commission européenne ait cessé, comme cela était attendu, à la fin de l'année 2001, les participants ont décidé de poursuivre, chacun de leur côté, les recherches entreprises sur le patrimoine hospitalier, de s'efforcer de conserver les liens tissés à l'occasion du travail effectué en commun et, dans la mesure du possible, de continuer à alimenter le site web.

\section{NOTES}

1. Assistance publique-Hôpitaux de Paris. Patrimoine hospitalier, un parcours à travers l'Europe. Paris : Editions du patrimoine, 2001.64 p.

\section{INDEX}

Mots-clés : France, Italie, Angleterre, architecture hospitalière, inventaire du patrimoine Keywords : architectural heritage

\section{AUTEUR}

\section{PIERRE-LOUIS LAGET}

Conservateur du patrimoine, Service régional de l'Inventaire, Direction régionale des affaires culturelles du Nord-Pas-de-Calais. pierre-louis.laget@culture.gouv.fr 